Unfortunately, our attempt to investigate the effect of $M$ on these relationships was not successful, as we found no effect of $M$ of the $S-R$ pair on recall probability in this experiment. Treating as $M$ the association value (AV) of the stimulus-response pair as a trigram (Witmer, 1935), the correlation of proportion correct and AV was only .02 . We attempted further to correlate proportion correct on an item with the normative frequency with which the third letter or S-term (Underwood \& Schulz, 1960. p. 374). Despite wide variation of this measure in our sample, it accounted for practically none of the variation across items in the proportion correct $(r=.11$, $\mathrm{p}>.10)$.

\section{RLFERENCLS}

BERNBACH, H. A. Decision processes in memory. Psychological Review, 1967, 74, $462-480$.

MURDOCK, B, B., JR. The criterion problem in short-term memory. Journal of Experimental Psychology, 1966, 72, 317-324.

SUBOSKI, M D. PAPPAS, B. B., \& MURRAY, D. J. Confidence ratings in recall paired-associates learning. Psychonomic Science, 1966, 5, 147-148.

UNDERWOOD, B. J., \& SCHULZ, R. W. Meaningfulness and verbal learning. New York: Lippincott, 1960.

WITMER, $L$. $R$. The association value of three-place consonant syllables. Journal of Genetic Psychology, 1935, 47, 337-360.

\title{
Recency/primacy ratio: A short test of task orientation
}

\section{PETER HAMILTON \\ Medical Research Council Applied Psychology Unit, Cambridge, England and \\ G. R. J. HOCKEY \\ University of Durham, Durham, England}

Thirteen Ss recalled 60 nine-digit lists presented consecutively for $15 \mathrm{~min}$ at a rate of two digits/second. An analysis of errors by serial position revealed that (1) primacy errors in the first two list positions increased over time, and (2) recency errors in the last two list positions decreased over time. The ratio recency errors/primacy errors showed a consistent downward trend over the testing period. It is suggested that this ratio is an index of the degree of active information processing and may be a useful reflection of the vigilance type of decrement, which can be derived from the use of a short simple test.

The measurement of performance of the individual under conditions of stress, prolonged work, and diverse affective states is often frustrated by the lack of sensitive tests. A major criticism seems to be task duration, only relatively long tasks being successful in reflecting such changes in performance as occur under these conditions. Broadbent (1958) and Wilkinson (1968), for example, list the desirable characteristics of tests for measuring effects of noise and sleep loss, respectively; task duration is regarded as of primary importance in both cases. Short tes: are generally not sensitive to such changes in the state of the individual as can readily be achieved in the laboratory, though, in real-life situations (such as clinical diagnoses and industrial measurement), the considerable time pressures involved make the development of short sensitive tests highly desirable.

Two broad categories of "traditional" tests of such performance changes can be distinguished. (1) Perceptual-motor, "information-throughput" tasks, such as tracking, and the five-choice serial reaction task (Broadbent, 1963); performance decrement in these tests appears as an increase in either the variance or the length of response times over a $1 / 2 \mathrm{~h}$ or so, or as an increase in tracking error, such as time-off-target. (2) Vigilance tests (Davies \& Tune, 1970); here, degradation takes the form of an accelerated decrement in detection probability over time or of a general lowering of detection probability. Because of the wide range of individual variation on both kinds of test, a repeated testing design is usually necessary to establish reliability.

The present experiment is motivated by the need for simple "one-shot" tests of performance, applicable to group testing situations. The minimum criteria for such a test are that it is sensitive to the typical decrement with time on task, in a short time period, and in a single testing session. An additional desirable aspect of such a test is that it should be portable and simple to administer (the kinds of tasks described above often require considerable practice because of the unusual nature of the task demands).

Auditory short-term memory, in which 8-10 digits or letters are presented sequentially, possesses the basic qualities of such a test, though, as yet, it has not been used to study performance change over a period of repeated presentations. Performance on such tests is characterized by a pronounced serial-position curve of error. Various theories (Broadbent, 1958; Waugh \& Norman, 1965; Glanzer \& Cunitz, 1966) have attributed the shape of the curve to two components: one, a very short-term "echo-box" store with a time constant of a few seconds, which ensures efficient recall of the last few items (recency); the other, a longer-term store which is assumed to be the source of high recall of the first few items input (primacy). It is likely that the extent of the primacy effect is dependent on the rate of information processing during the input of the list. Crowder (1969) found that the primacy effect disappeared on the standard nine-digit list he was studying when he presented Ss with a series of lists of different length. He attributes this effect to lack of active rehearsal of the first few items-the $S$ anticipating a much longer list.

If the reduction in active information processing seen in the Crowder paradigm is comparable with that occurring when decrement is observed in perceptual-motor tasks or vigilance situations, we should anticipate a loss of primacy in a condition that requires continuous memorizing and recall of short lists. Moreover, the amount of data to be obtained in a short period with such a test should ensure a high degree of reliability within an economical experimental paradigm.

\section{SUBJECTS}

Thirteen adult males and females were paid to attend the session.

\section{DESIGN AND PROCEDURE}

Ss were presented with 60 nine-digit lists and asked to recall each immediately after it occurred. Within each list, digits were presented at two/second, synchronized with metronome beats. An interval of
Table 1 Mean Percent Error at Each List Position

\begin{tabular}{lrrrrrrrrr}
\hline Position & 1 & 2 & 3 & 4 & 5 & 6 & 7 & 8 & 9 \\
\hline $\begin{array}{l}\text { Percent } \\
\text { Error }\end{array}$ & 5.0 & 22.4 & 38.7 & 46.0 & 58.5 & 66.7 & 65.9 & 50.3 & 13.5 \\
\hline
\end{tabular}

Table 2

Mean Percent Correct Recall Over Six Blocks of 10 Lists

\begin{tabular}{lcccccc}
\hline Block & 1 & 2 & 3 & 4 & 5 & 6 \\
\hline $\begin{array}{l}\text { Percent } \\
\text { Correct }\end{array}$ & 58.0 & 60.0 & 56.7 & 57.5 & 62.9 & 60.7 \\
\hline
\end{tabular}




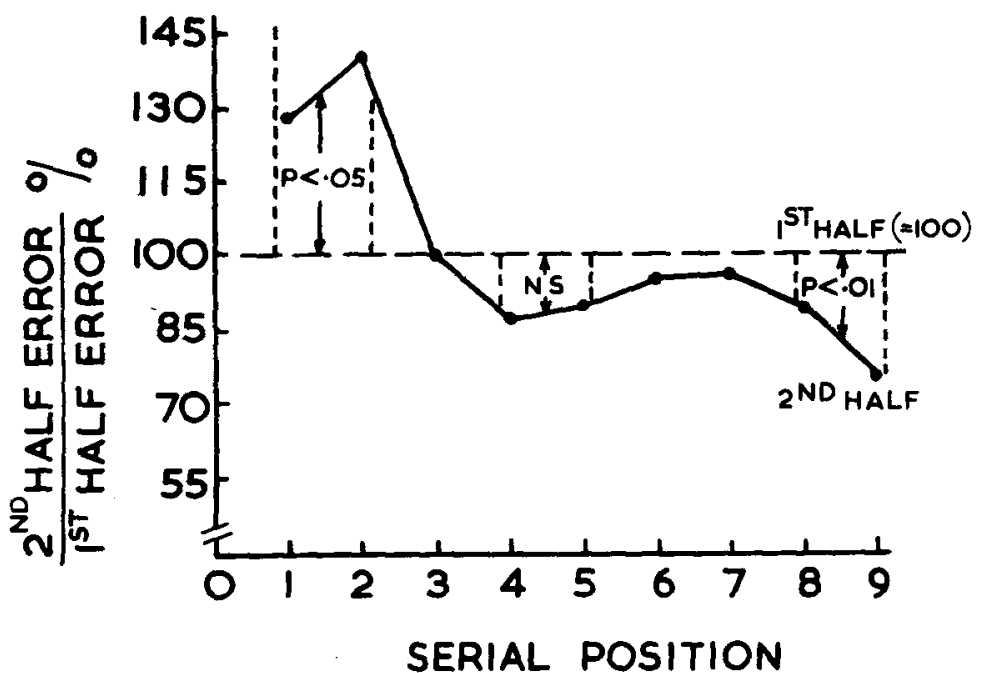

Fig. 1. Errors at each serial position in the second half of the test as a percentage of first-half errors.

$10.5 \mathrm{sec}$ preceded the onset of the next list, which was announced by four metronome beats; list-to-list cycle was $15 \mathrm{sec}$. The whole task, therefore, lasted $15 \mathrm{~min}$. Each list exhausted the set of digits 1 to 9 . Ss were instructed to recall the digits in order and to leave blanks if they were unsure as to the digit in a specific position.

\section{RESUITS AND DISCUSSION}

The mean percent error rate for each serial position is shown in Table 1 .

Table 2 shows the mean number of digits recalled in correct position for each of six blocks of 10 lists. No overall decrement was observed over the task duration.

Results were analyzed by serial position and the first block of 30 lists compared with the second. In Fig. 1 the error score for each of the nine positions in the second half of the test is expressed as a percentage of scores in the first half. The number of errors apparently increases on the first two positions but decreases slightly in the

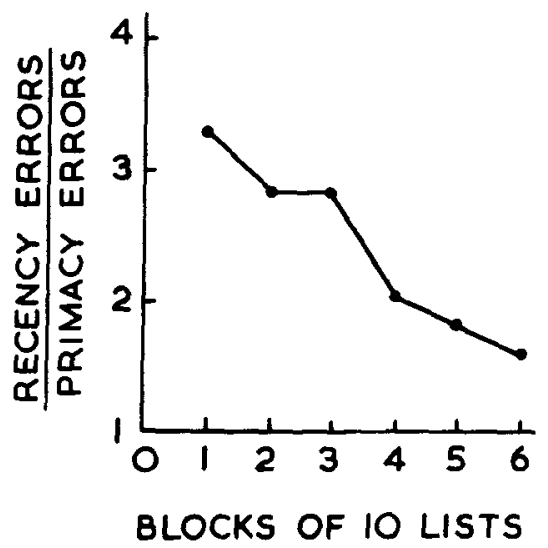

Fig. 2. Recency/primacy ratio as a function of time on task. middle and to a greater extent in the recency region. Wilcoxon t tests between halves were carried out on Positions 1 and 2,4 and 5 , and 8 and 9 . The probability levels associated with these tests are shown in Fig. 1.

There was a significant change in performance in two respects: an unexpected decrease in errors in the recency region and the predicted increase in errors in the primacy region. Accordingly, an index of performance in terms of recency errors/primacy errors provides a measure presumably sensitive to change over time. Figure 2 shows the time course of this index over the six blocks of 10 lists; the change in this index is apparent. Dividing the test into three blocks of 20 lists, a Friedman two-way analysis of variance for the index is significant $(p<.05)$. Twelve of the $13 \mathrm{Ss}$ show a decrease in the ratio from the first 20 lists to the last 20.

Recency/primacy ratio thus seems to provide (at least for nine-digit lists) a highly sensitive index of a factor which is here termed task orientation. It is assumed that what is measured is some reflection of the degree to which $S$ indulges in active processing of the input, as opposed to passive listening. An alternative explanation of the effect would be in terms of proactive interference (PI) between lists. If PI builds up during the session and if it affects only the primacy region of the list, primacy scores would decline while recency scores might benefit from a lack of response interference. This explanation is unlikely to be correct by virtue of the lack of decrement (and even a slight increase) in efficiency at Positions $3,4,5$, and 6 , which are generally deemed to be in the primacy region. It seems more probable that a lowered level of processing activity on the first part of the list would allow some reduction in within-list $P I$ to benefit these later primacy items. Furthermore, the hypothesis that performance on the last two positions benefits from a reduction of response interference from earlier items can be tested by comparing the number of responses made to Positions 1.7 in the first and second halves of the test. The mean number of responses made in the first half was $187.5(89.3 \%)$ and in the second 185.5 $(88.3 \%)$. The difference is not significant and it is concluded that no significant reduction in response interference occurred.

Future use of this test will concentrate on assessment of effects of various environmental stressors on performance and of differences due to factors such as age or personality. The measure will obviously have greater sensitivity where within-Ss comparisons are being made or where independent groups are previously matched on some relevant criterion, such as intelligence. Occasionally, Ss will be tested who make no errors in the primacy region. These must either be rejected or some reference error rate for the primacy area applied throughout the group (e.g., each $S$ is given one error in 10 trials and their personal error added to this). An alternative measure is the number of recency errors expressed as a ratio of the total number of recency plus primacy errors. This gives a ratio of 1 for cases where no primacy errors occur, but still does not discriminate between high- and low-recency scores in these instances. Here again a reference error rate is required.

The use of this test would not mean a replacement of the more traditional measures of performance change with time, since these may well provide more specific and useful information. It does, however, suggest a convenient alternative in situations where time and research effort are limited.

\section{REFERENCES}

BROADBENT, D, E. Perception and communication. London: Pergamon, 1958.

BROADBENT, D. E. Differences and interactions between stresses. Quarterly Journal of Experimental Psychology, 1963, 15, 205-211.

CROWDER, R. G. Behavioral strategies in immediate memory. Journal of Verbal Leaming \& Verbal Behavior, 1969, 8, 524-528.

DAVIES, D、R., \& TUNE, G. S. Human vigilance performance. London: Staples Press, 1970.

GLANZER, M, \& CUNITZ, A. R. Two storage mechanisms of free recall. Journal of Verbal Learning \& Verbal Behavior, 1966, 5, 351-360.

WAUGH, N. C., \& NORMAN, D. A. Primary memory. Psychological Review, 1965, 72, 89-104.

WILKINSON, R. T. Sleep deprivation: Performance tests for partial and selective sleep deprivation. In L. E. Abt and B. F. Reiss (Eds.), Progress in clinical psychology. New York: Grune \& Stratton, 1968. 